\title{
Recent Results From the Compton@HIS Collaboration
}

\author{
Mark Sikora* and the Compton@HI $\gamma$ S Collaboration \\ George Washington University \\ E-mail: msikoradtunl.duke.edu
}

\begin{abstract}
The electromagnetic properties of the nucleon arise from its composite nature. External electric and magnetic fields induce dipole moments described by the scalar polarizabilities $\left(\alpha_{N}\right.$ and $\left.\beta_{N}\right)$, while the response of the nucleon spin is described by four spin polarizabilities. Consequently, studying these observables offers a window into the interaction among the constituent charges and currents in the nucleon, described theoretically by Quantum Chromodynamics (QCD). In particular, high-precision measurements of the scalar and spin polarizabilities provide a means for studying the low-energy, non-perturbative regime of QCD. Such measurements are needed to constrain emerging calculations from lattice QCD and Chiral Effective Field Theories and are critical inputs to descriptions of the proton-neutron mass difference. To this end, a program of Compton scattering experiments on light nuclei is underway at the High Intensity Gamma Source $(\mathrm{HI} \gamma \mathrm{S})$ at Duke University, with the aim of providing the world's most precise data to extract the polarizabilities.
\end{abstract}

The 9th International workshop on Chiral Dynamics

17-21 September 2018

Durham, NC, USA

\footnotetext{
${ }^{*}$ Speaker.
} 


\section{Introduction}

The response of the nucleon to external electric and magnetic fields arises due to its composite nature as a bound system of quarks and gluons governed by QCD and the electromagnetic force. It is encoded in the static electric $\left(\alpha_{N}\right)$ and magnetic $\left(\beta_{N}\right)$ dipole polarizabilities, which describe the stretchability of the nucleon charge distribution and the alignability of the magnetic constituents, and four spin polarizabilities which describe the response of the nucleon spin vector to applied fields. These quantities are explored experimentally using nuclear Compton scattering, in which the electric and magnetic fields of a real photon induce radiation multipoles by displacing charges and currents inside the nucleon.

As intrinsic, fundamental properties of the nucleon, the polarizabilities have been of interest to theoretical approaches to non-perturbative QCD. As improved $a b$ initio calculations from lattice QCD [1] and Chiral Effective Field Theories [2] emerge, precise experimental values for the polarizabilities are essential for providing stringent benchmarks. In addition, the polarizabilities have been shown to be key to theoretical treatments of muonic hydrogen [3] and the proton-neutron mass difference [4, 5], where the dominant source of uncertainty is the experimental error in $\beta^{(n)}$ of the neutron.

The polarizabilities appear at higher-order terms in the expansion of the Hamiltonian for nuclear Compton scattering. At first order in photon energy,

$$
H_{e f f}^{(1)}=\frac{-e(1+\kappa)}{2 m} \vec{\sigma} \cdot \vec{H}-\frac{e(1+2 \kappa)}{8 m^{2}} \vec{\sigma} \cdot[\vec{E} \times(\vec{p}-e \vec{A})-(\vec{p}-e \vec{A}) \times \vec{E}]
$$

where $\vec{A}$ is the vector potential, $\kappa$ is the anomalous magnetic momenent of the nucleon and $\vec{p}$ is its momentum. The internal degrees of freedom of the nucleon contribute at the next highest-order term in the expansion

$$
H_{e f f}^{(2)}=-4 \pi\left[\frac{1}{2} \alpha_{E 1} \vec{E}^{2}+\frac{1}{2} \beta_{M 1} \vec{H}^{2}\right]
$$

where $\alpha_{E 1}$ and $\beta_{M 1}$ are the scalar polarizabilities. The next highest-order term contains the four spin polarizabilities denoted by $\gamma$

$$
H_{e f f}^{(3)}=-4 \pi\left[\frac{1}{2} \gamma_{E 1 E 1} \vec{\sigma} \cdot(\vec{E} \times \dot{\vec{E}})+\frac{1}{2} \gamma_{M 1 M 1} \vec{\sigma} \cdot(\vec{H} \times \dot{\vec{H}})-\gamma_{M 1 E 2} E_{i j} \sigma_{i} H_{j}+\gamma_{E 1 M 2} H_{i j} \sigma_{i} E_{j}\right]
$$

where the subscripts refer to the multipolarity of the incoming and scattered photons respectively.

The status of the scalar polarizabilities from EFT is summarized in Fig. 16]. The diagonal bands are constraints imposed by the Baldin Sum Rule, which relates the sum of $\alpha$ and $\beta$ to the total photoabsorption cross section

$$
\alpha+\beta=\frac{1}{2 \pi^{2}} \int_{\omega_{t h}}^{\infty} \frac{\sigma_{\gamma}(\omega) d \omega}{\omega^{2}} .
$$

The most recent extraction of $\alpha$ and $\beta$ gives the following values for the proton and neutron:

$$
\begin{array}{ll}
\alpha_{E 1}^{(\mathrm{p})}=10.65 \pm 0.35_{\text {stat }} \pm 0.2_{\text {Baldin }} \pm 0.3_{\text {th }}, & \beta_{M 1}^{(\mathrm{p})}=3.15 \mp 0.35_{\text {stat }} \pm 0.2_{\text {Baldin }} \mp 0.3_{\text {th }} \\
\alpha_{E 1}^{(\mathrm{n})}=11.55 \pm 1.25_{\text {stat }} \pm 0.2_{\text {Baldin }} \pm 0.8_{\text {th }}, & \beta_{M 1}^{(\mathrm{n})}=3.65 \mp 1.25_{\text {stat }} \pm 0.2_{\text {Baldin }} \mp 0.8_{\text {th }}
\end{array}
$$




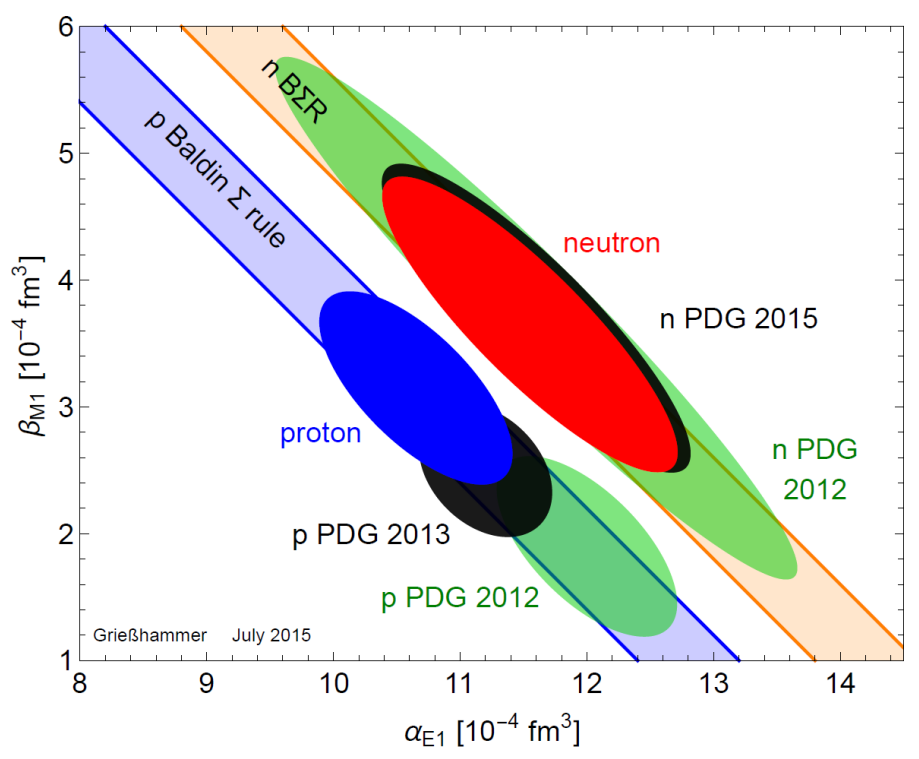

Figure 1: Status of the scalar polarizabilities, as reported in the Particle Data Group (PDG). The diagonal bands indicate parameter space allowed by the Baldin Sum Rule. Figure from Ref. [6].

Of particular note is the substantial statistical uncertainty of $\sim 30 \%$ for $\beta_{M 1}^{(\mathrm{n})}$ compared to the relatively well-known proton values. This is a reflection of the difficulty in obtaining high statistics Compton scattering data for the neutron. Due to the lack of a free neutron target, this requires the use of light nuclei like deuterium, which presents experimental challenges due to a Compton scattering cross section on the order of nanobarns and which is only weakly bound. Consequently, identifying elastic scattering events from photodisintegration events presents an additional difficulty.

The spin polarizabilities can be thought of as a parameterization of the stiffness of the nucleon spin vector to precession in applied magnetic fields, similar to the classical Faraday effect. They are accessed experimentally by measuring polarization observables using combinations of circularly and linearly polarized photon beams and targets which are longitudinally or transversely polarized or even unpolarized. These observables then constrain the reaction amplitudes for Compton scattering from which the spin polarizabilities are extracted. The first experimental values for the spin polarizabilities of the proton were obtained from experiments at Mainz utilizing a polarized target (in $10^{-4} \mathrm{fm}^{4}$ ) [7]

$$
\gamma_{E 1 E 1}=-3.5 \pm 1.2, \quad \gamma_{M 1 M 1}=3.2 \pm 0.9, \quad \gamma_{E 1 M 2}=-0.7 \pm 1.2, \quad \gamma_{M 1 E 2}=2.0 \pm 0.3
$$

To expand the global database of polarized Compton scattering, a measurement using a linearly polarized beam on an unpolarized ${ }^{1} \mathrm{H}$ target was planned at Mainz in order to measure the beam asymmetry $\Sigma_{3}$ defined as the asymmetry between scattering parallel and perpendicular to the plane of incident beam polarization

$$
\Sigma_{3}=\frac{\sigma^{\|}-\sigma^{\perp}}{\sigma^{\|}+\sigma^{\perp}}
$$

The beam asymmetry is most sensitive to $\gamma_{E 1 E 1}$ and $\gamma_{M 1 M 1}$. This observable has the added feature of facilitating an extraction of $\beta_{M 1}$ which does not require imposing the Baldin Sum Rule 
constraint [8].

\section{The Compton@HI $\gamma$ S Program}

In response to these challenges, the Compton@HI $\gamma \mathrm{S}$ program was developed. This program seeks to exploit the unique characteristics of the beams produced at the HI $\gamma \mathrm{S}$ facility [9] to perform Compton scattering measurements on a series of light nuclei with high statistical accuracy. These include a commissioning run using ${ }^{4} \mathrm{He}$ to test the new apparatus, Compton scattering measurements on ${ }^{2} \mathrm{H}$ with circularly polarized beams at 65 and $85 \mathrm{MeV}$ to study the neutron magnetic polarizability $\beta_{n}$, and a measurement of the beam asymmetry $\Sigma_{3}$ of Compton scattering on ${ }^{1} \mathrm{H}$ at $84 \mathrm{MeV}$ to study the spin polarizabilities.

The HI $\gamma \mathrm{S}$ beam is produced via Compton backscattering from a free electron laser. The resulting gamma-ray beam is quasi-monoenergetic with $100 \%$ circular or linear polarization. A beam burst is delivered to the target area every $179 \mathrm{~ns}$, and this time structure is essential to event identification. Intensities on the order of $10^{7} \gamma / \mathrm{s}$ were achieved during the experiments presented here. Such high intensities are critical to achieving the count rates necessary to measure nanobarn cross sections in a reasonable amount of beam time while maintaining a signal-to-noise ratio high enough to obtain the desired statistical uncertainties. In order to maximize the intensity, a $25 \mathrm{~mm}$ diameter collimator was used, which increased the intensity at the expense of broadening the energy distribution of the beam to $15 \%$ full-width at half-maximum (FWHM). At the time, this was a reasonable trade-off to make, as the observables of interest do not vary significantly over such an energy spread. However, for the measurements on deuterium, this clearly exceeds the $2.25 \mathrm{MeV}$ binding energy, but the existing detectors were not capable of resolving inelastic scattering from photodistintegration in any case. This complication will be addressed in the next sections.
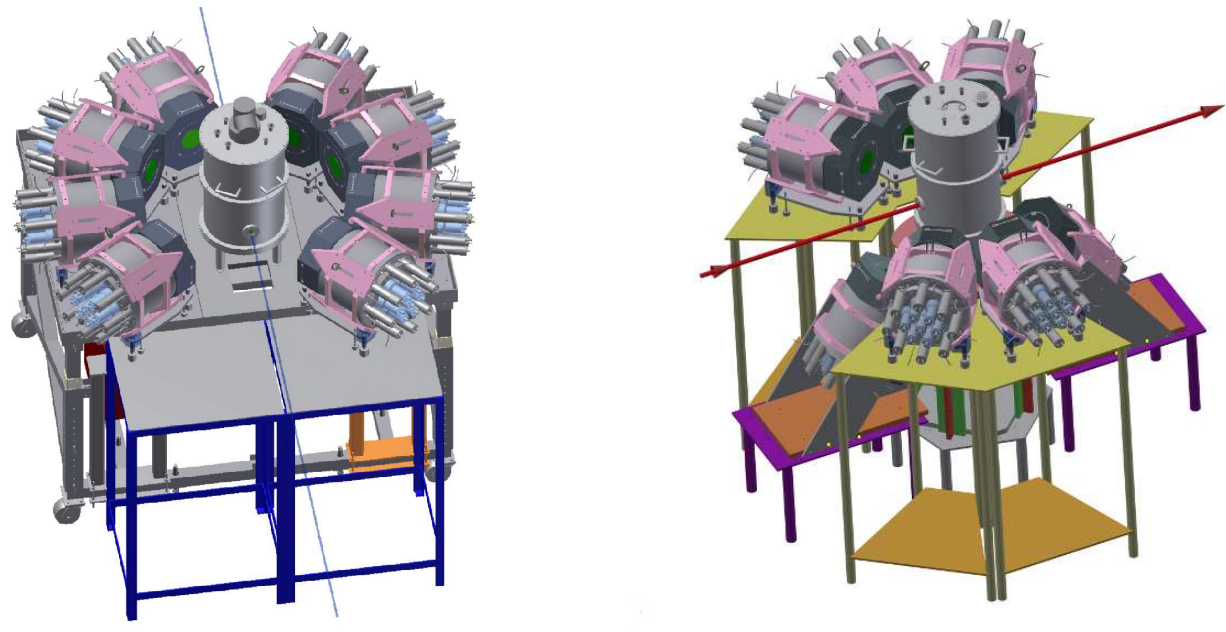

Figure 2: Experimental setup showing the cryotarget and HINDA detectors, with the beam direction indicated. The configuration on the left was used for the experiments on ${ }^{4} \mathrm{He}$ and ${ }^{2} \mathrm{H}$, which used a circularly polarized beam and all detectors in-plane. The configuration on the right was used for the beam asymmetry measurement with horizontal linear polarization, where three detectors are placed out of plane.

Besides intense beams, the count rate requirements for these experiments require liquid rather than gas targets. To this end, a cryogenic system capable of liquefying hydrogen, deuterium, and 
${ }^{4} \mathrm{He}$ was designed and built [10]. The liquid is contained in a $0.13 \mathrm{~mm}$ thick Kapton cell and is free of visible bubbles. The target sits in the center of an array of eight $10^{\prime \prime} \times 10^{\prime \prime} \mathrm{NaI}$ detectors collectively known as the HI $\gamma \mathrm{S}$ NaI Detector Array (HINDA). Each HINDA detector is surrounded by a segmented $\mathrm{NaI}$ anti-coincidence shield to assist in cosmic-ray rejection. Fig. 2 shows the two configurations used for the present experiments. The circularly polarized measurements on ${ }^{4} \mathrm{He}$ and ${ }^{2} \mathrm{H}$ had all detectors arranged in-plane, while 3 detectors were placed on the floor for the beam asymmetry measurement.
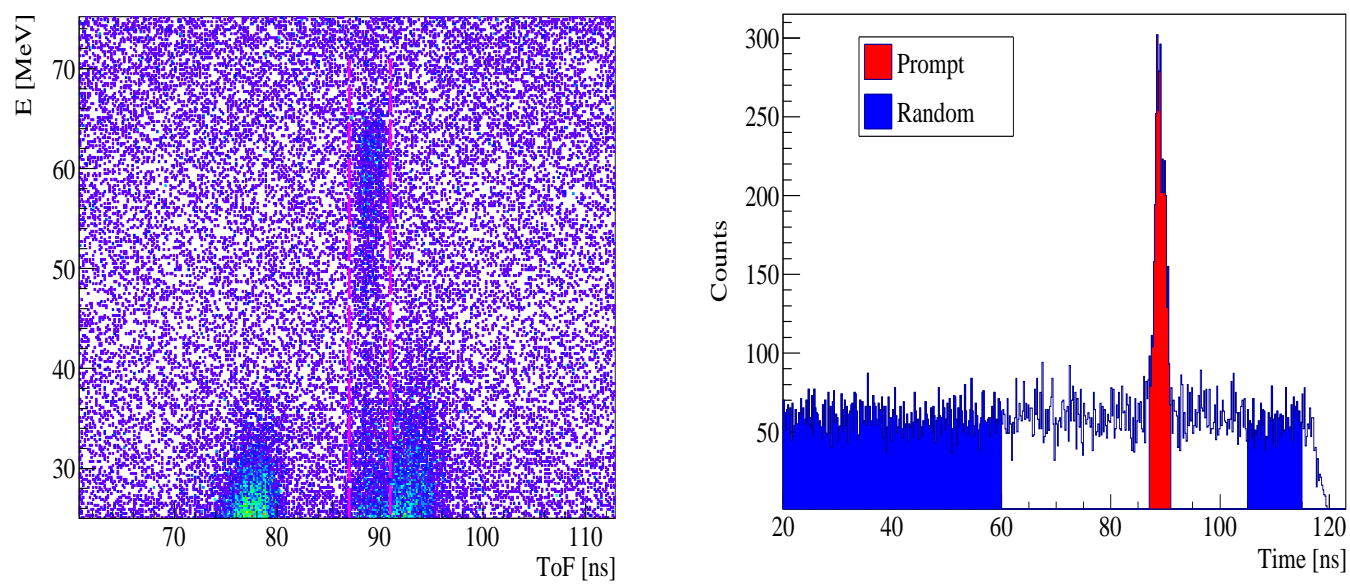

Figure 3: (Left) Compton-scattered events are identified by plotting detector energy vs. event time with respect to the beam pulse. A timing window can then be set. (Right) Projecting the time axis, after applying an energy cut shows a prominent prompt timing peak for beam-related events.

The energy region of interest is dominated by cosmic-ray events, which occur at a rate three orders of magnitude greater than Compton-scattered events but which are not correlated with the beam burst. The Compton events can be identified using a scatter plot of detected energy and event time with respect to the constant $5.56 \mathrm{MHz}$ frequency of the beam, as can be seen in Fig. 3 where Compton-scattered events are clearly distinguished. Projecting this spectrum onto the time axis above a threshold energy shows a prominent prompt-timing peak for beam-related events while non-beam-related events have a flat, random timing distribution. The uncorrelated background consists primarily of comsic-ray events, some of which occur within the prompt timing peak. The anti-coincidence shields surrounding each HINDA detector can be used to suppress this background by vetoing events which leave a large energy signature in the shields. Events from the prompt and random regions are then sampled, and the randoms contribution is scaled and subtracted.

The resulting energy spectra are shown in Fig. 4 at both forward and backward angles. Forwardangle spectra include an exponential low-energy background from atomic scattering, which is kinematically suppressed at backward angles, which are background-free. Compton scattering lineshapes are obtained using a simulation of the detector response in Geant 4 which is then fit to the scattering spectra simultaneously with the background functions. The lineshape fits can then be used to define integration windows to extract the cross section. 

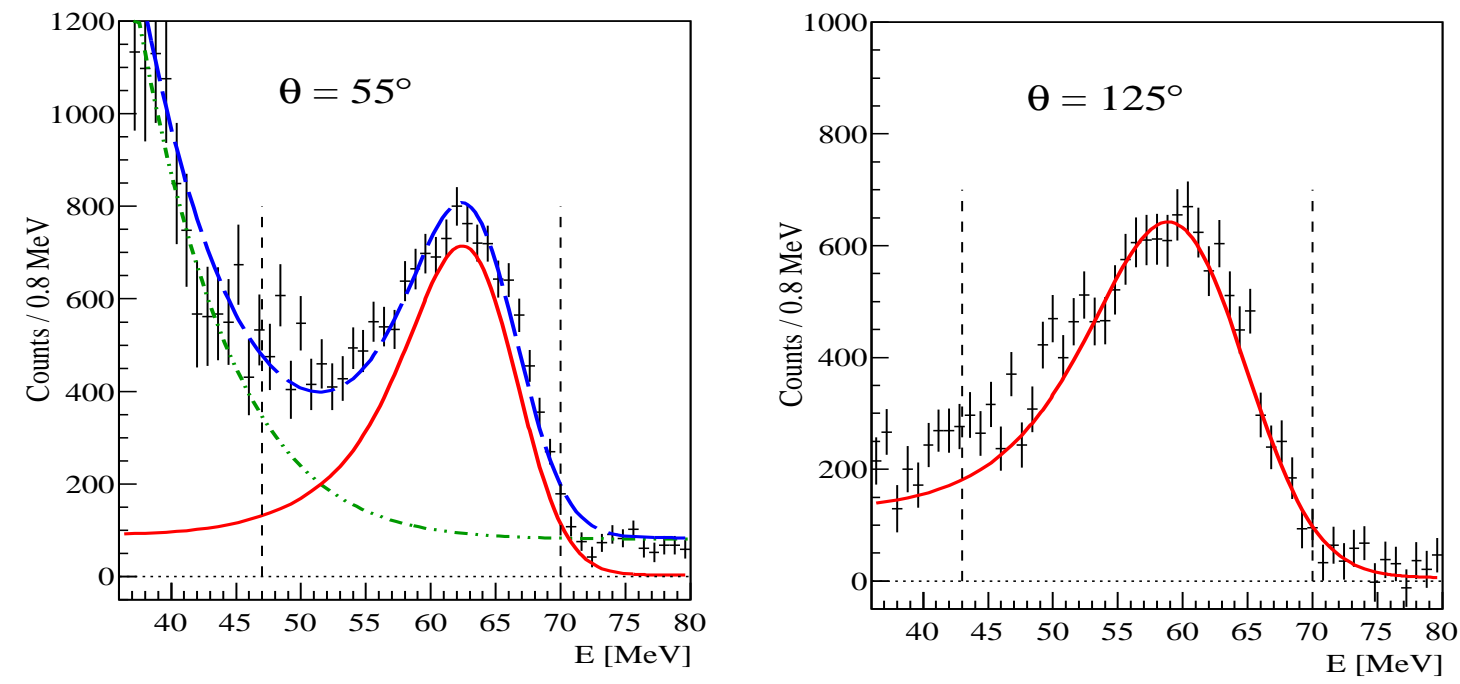

Figure 4: Examples of energy spectra at a forward and backward angle, along with lineshape and background fits.

\section{Preliminary Results}

The apparatus was commissioned using ${ }^{4} \mathrm{He}$ as the target nucleus and a circularly polarized $61 \mathrm{MeV}$ gamma-ray beam. Intended as a commissioning exercise prior to performing longer measurements requiring several hundred hours, the results shown in Fig. 5 were obtained in only $\sim 54$ hours of beam time [11]. The consistency of the new data with the only other measurements at this beam energy [13] provided validation of the present experimental setup.

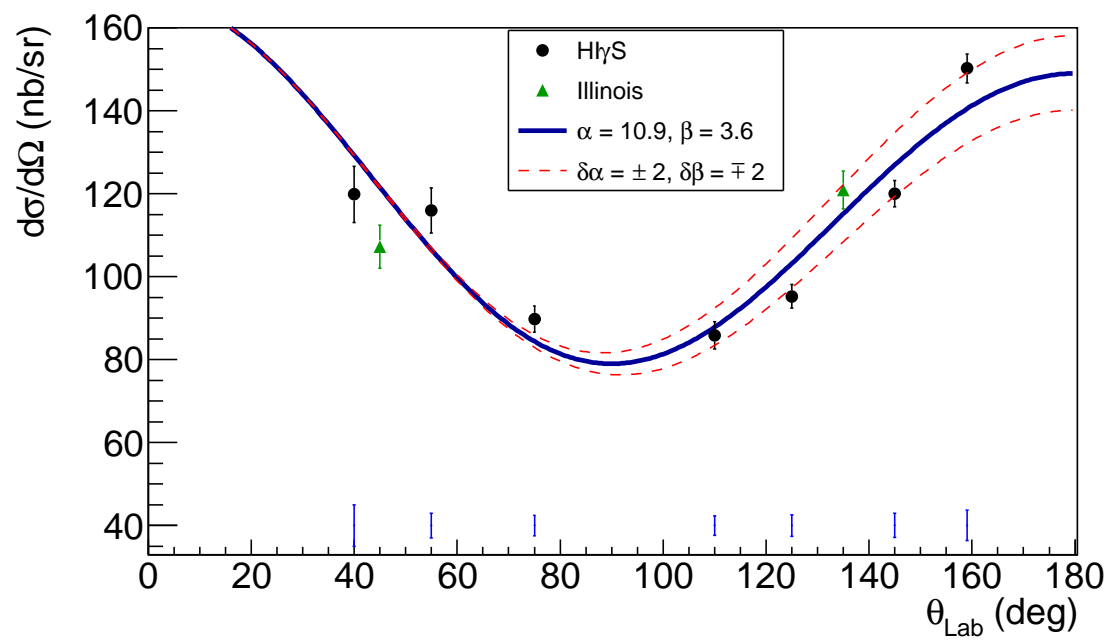

Figure 5: ${ }^{4} \mathrm{He}$ Compton scattering angular distribution at $\mathrm{E}_{\gamma}=61 \mathrm{MeV}$, compared with unpublished data taken at the University of Illinois [13]. The potential sensitivity of the data to the polarizabilities is assessed using the phenomenological model described in [12]. The systematic uncertainties are shown beneath each data point. 
Despite the absence of a full EFT treatment, some conclusions about the data can be drawn using a phenomenological model based on the total photoabsorption cross section [12]. The inputs to the model are taken from fits of the E1 strength and quasi-deuteron cross section from fits of ${ }^{4} \mathrm{He}$ photoabsorption [14], and the isospin-averaged nucleon polarizabilities $\alpha_{S}$ and $\beta_{S}$ were taken as the accepted free-nucleon values from EFT. The model curves shown in Fig. 5 then do not include any free parameters. The data are well-described by the model at this beam energy. The dotted curves show the effect of varying $\beta_{S}$ by \pm 2 units while keeping the sum-rule constraint $\alpha_{S}+\beta_{S}=14.5$ intact. This preserves the forward-angle cross section, while the resulting change in $\alpha_{S}-\beta_{S}$ varies the back-angle cross section. As shown in Fig. 5, the predicted sensitivity to $\alpha_{S}-\beta_{S}$ exceeds the statistical uncertainties of $2-4 \%$ in this region. The consistency of the new data with the phenomenological model with the sum-rule constraint confirms the analysis procedure and our absolute normalization.

After establishing the validity of the new apparatus, two measurements on deuterium were performed with circularly polarized beams at energies of 65 and $85 \mathrm{MeV}$. Approximately 300 hours of beam on target was collected at each energy. As mentioned previously, the large collimator diameter used in the production of these beams results in a beam-energy spread of $\sim 15 \%$ FWHM, which at these energies exceeds the $2.25 \mathrm{MeV}$ binding energy of the deuteron. Consequently, the preliminary cross sections shown in Fig. 3 contain both elastically scattered Compton events and inelastically scattered photodisintegration events. Kinematically, the inelastic contribution is expected to increase with increasing momentum transfer, which occurs at more backward angles. In the top panel in Fig. 3, the most forward-angle data point coincides with the EFT calculation of pure elastic scattering. Scanning across the measured angular range, the deviation of the data from the expected Compton scattering cross section is seen to increase with angle due to the inelastic contribution. This back-angle discrepancy is enhanced at the higher beam energy, shown in the bottom panel of Fig. 3, where now a small deviation is also observed at forward angles as well due to the increase in beam energy.

The effect of these data on the polarizabilties must await corrections accounting for the inelastic contribution, plans for which will be discussed in the next section. Nevertheless, comparisons to existing EFT calculations [15] at each beam energy demonstrate once again the sensitivity achievable with high statistical accuracy datasets. The back-angle data have experimental uncertainties of $\sim 5 \%$ or better. Even after correcting for the inelastic contribution, these data should be the world's most accurate measurement of Compton scattering on deuterium.

The most recent experiment completed in the Compton@HI $\gamma \mathrm{S}$ program is the beam asymmetry measurement on the proton using a linearly polarized $84 \mathrm{MeV}$ beam. Three of the HINDA detectors were placed on the floor in order to measure scattering in the plane perpendicular to the plane of incident polarization (see Fig. 2). The relative solid angles between the perpendicular and parallel planes were normalized by first taking 200 hours of scattering data using a circularly polarized beam. This was followed by 150 hours of linearly polarized beam on target, and the preliminary beam asymmetries are shown in Fig. 7 .

Previously published data from Mainz [16] and a calculation from heavy baryon chiral perturbation theory (HB $\chi \mathrm{PT})$ [17] are available for comparison. The new data complement the wide angular coverage achievable with the Crystal Ball detector at Mainz by measuring a narrower set of angles with a factor of $\sim 2$ improvement in statistical uncertainty. The new data are consis- 

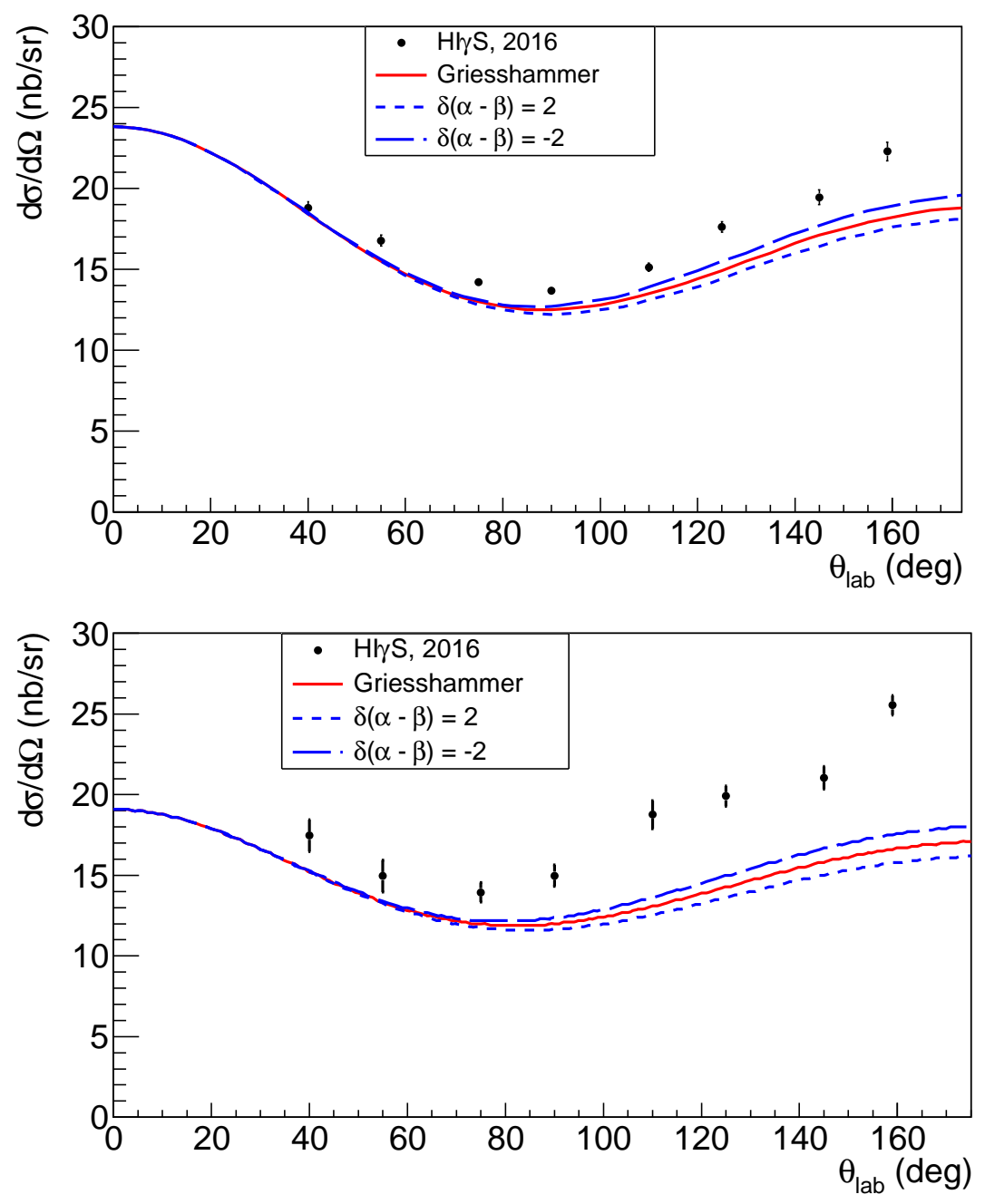

Figure 6: (Top) Preliminary angular distribution obtained on ${ }^{2} \mathrm{H}$ at $65 \mathrm{MeV}$. (Bottom) Preliminary angular distribution obtained on ${ }^{2} \mathrm{H}$ at $85 \mathrm{MeV}$. Note that in both plots, the data include both elastic (Compton) and inelastic scattering, while the EFT calculations pertain only to elastic Compton scattering [15].

tent with existing measurements, and both experiments report a smaller asymmetry at $90^{\circ}$ than the theoretical prediction, which is maximal near this angle. Analysis of these data is ongoing.

\section{Outlook}

In addition to the work described here, the Compton@HI $\gamma \mathrm{S}$ program includes two more approved experiments to be run in the near future. As presented above, the Compton scattering data collected on deuterium includes both elastic and inelastic scattering events. In order to report a pure elastic scattering result, from which the polarizabilities can be extracted, the inelastic contribution must be characterized. To this end, an experiment to measure this contribution directly has been proposed and approved at HI $\gamma \mathrm{S}$ [19]. To achieve the necessary energy resolution in the scattering spectra, a narrow collimator will minimize the energy spread of the beam, and two high-resolution 


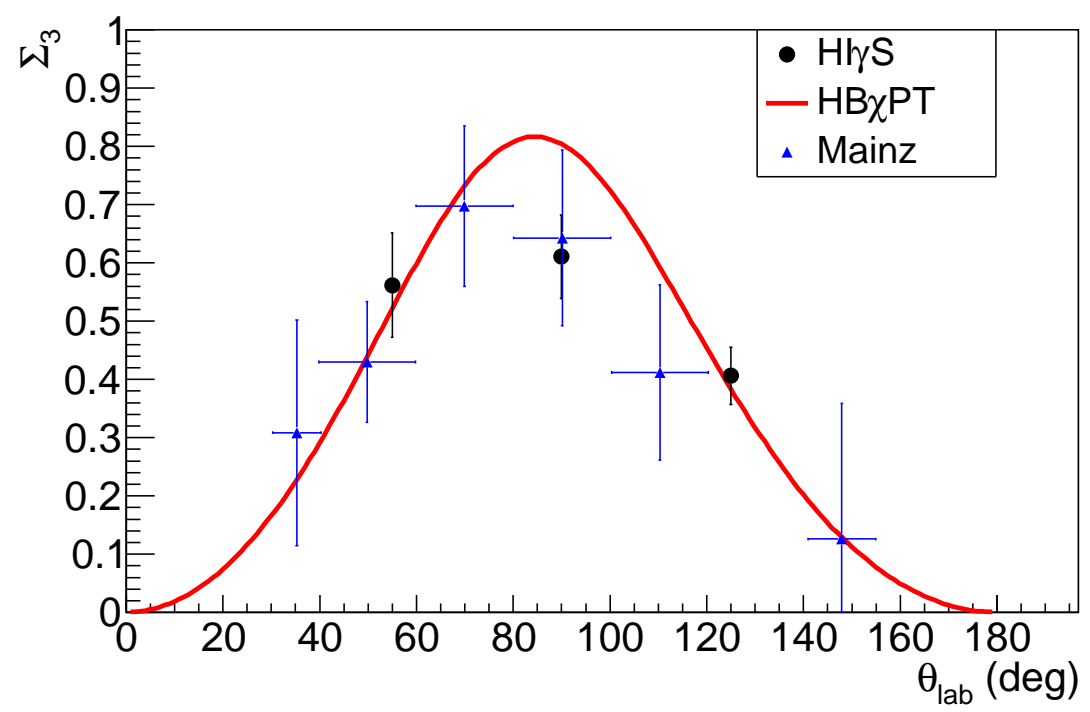

Figure 7: Preliminary beam asymmetry measurements at $\mathrm{E}_{\gamma}=84 \mathrm{MeV}$. Results from Mainz [16] and a theoretical calculation [17] are shown for comparison.

( $\sim 2 \%$ at $100 \mathrm{MeV}$ ) NaI detectors will be used in place of HINDA. Further validation of results from such an experiment can come from theoretical predictions from calculations of quasi-free Compton scattering.

A future experiment will also perform the first ever measurement of Compton scattering from ${ }^{3} \mathrm{He}$ to obtain information on the isoscalar polarizabilities [18]. The choice of ${ }^{3} \mathrm{He}$, with $\mathrm{Z}=2$, offers a higher count rate relative to the $\mathrm{Z}=1$ targets, while inelastic scattering is separated from the Compton peak by $5.5 \mathrm{MeV}$ and thus resolvable using HINDA. Progress in EFT has reached a point where calculations on ${ }^{3} \mathrm{He}$ are available, so the prospect of a new data set with high statistical accuracy presents a unique opportunity. Modifications to the cryogenic target required for liquefying and operating with ${ }^{3} \mathrm{He}$ are ongoing.

\section{References}

[1] E. Change, S.R. Beane et al., Magnetic structure of light nuclei from lattice QCD, Phys. Rev. D 92 (2015) 114502.

[2] H.W. Grießhammer, J.A. McGovern, and D.R. Phillips, Nucleon polarisabilities at and beyond physical pion masses, Eur. Phys. J. A 52 (2016) 139.

[3] R. Pohl, R. Gilman, G.A. Miller and K. Pachucki, Muonic hydrogen and the proton radius puzzle, Ann. Rev. Nucl. Part. Sci., 63 (2013) 175.

[4] A. Walker-Loud, C.E. Carlson and G.A. Miller, Electromagnetic Self-Energy Contribution to $M_{p}-M_{n}$ and the Isovector Nucleon Magnetic Polarizability, Phys. Rev. Lett. 108 (2012) 232301.

[5] J. Gasser, M. Hoferichter, H. Leutwyler and A. Rusetsky, Cottingham formula and nucleon polarisabilities, Eur. Phys. J. C 75, no. 8, (2015) 375. 
[6] H.W. Grießhammer, J.A. McGovern, and D.R. Phillips, Compton Scattering and Nucleon Polarisabilities in Chiral EFT: The Next Steps, in proceedings of Chiral Dynamics 2015, PoS(CD15)036 (2015).

[7] P.P. Martel et al., Measurements of Double-Polarized Compton Scattering Asymmetries and Extraction of the Proton Spin Polarizabilities, Phys. Rev. Lett. 114 (2015) 112501.

[8] N. Krupina and V. Pascalutsa, Separation of proton polarizabilities with the beam asymmetry of Compton scattering, Phys. Rev. Lett. 110 (2013) 262001.

[9] H.R. Weller et al., Research opportunities at the upgraded HI $\gamma$ S facility, Prog. Part. Nucl. Phys., 62 (2009) 257.

[10] D.P. Kendellen et al., A cryogenic target for Compton scattering experiments at HI $\gamma$ S, Nucl. Instrum. Methods Phys. Res. Sect. A 840 (2016) 174.

[11] M.H. Sikora et al., Compton scattering from ${ }^{4} \mathrm{He}$ at $61 \mathrm{MeV}$, Phys. Rev. C 96 (2017) 055209.

[12] D.H. Wright et al., Elastic photon scattering from carbon and calcium and its interpretation, Phys. Rev. C 32 (1985) 1174.

[13] D. Wells, Ph.D. thesis, University of Illinois at Urbana-Champaign, Champaign, Illinois (1990).

[14] K. Fuhrberg, D. Häger, T. Glebe, et al., Compton scattering of $87 \mathrm{MeV}$ photons by ${ }^{4} \mathrm{He}$, Nuclear Physics A 591 (1995) 1.

[15] H.W. Grießhammer, private communication.

[16] V. Sokhoyan, et al., Determination of the scalar polarizabilities of the proton using beam asymmetry $\Sigma_{3}$ in Compton scattering, Eur. Phys. J. A, 53 (2017) 14.

[17] J.A. McGovern, D.R. Phillips, H.W. Grießhammer, Compton scattering from the proton in an effective field theory with explicit Delta degrees of freedom, Eur. Phys. J. A, 49 (2013) 12.

[18] G. Feldman, Compton scattering from ${ }^{3} \mathrm{He}, \mathrm{HI} \gamma \mathrm{S}$ PAC P-08-16.

[19] M.A. Kovash, M.W. Ahmed, Elastic and Inelastic Compton Scattering From Deuterium at 65 and 85 $M e V, \mathrm{HI} \gamma \mathrm{S}$ PAC P-13-17. 